This paper reported trends in mortality, incidence and prevalence of coronary heart disease (CHD), stroke, heart attack, angina and heart failure over the past 50 years.

Methods Mortality data were provided by the UK national statistics agencies. For morbidity data we reviewed the peer-reviewed and grey literature for comparable estimates from different time points over the last 50 years.

Results Around half of the UK population died from CVD in the 1960s; by 2009 this had dropped to a third. CHD mortality rates have remained 30\%-40\% higher in Scotland than in England since 1961. Incidence rates for heart attack have decreased since the 1960s, while survival has improved; prevalence in those over 75 has increased by around $40 \%$ since the mid-1990s. Over the past 20 years heart failure incidence decreased by over a third in Scotland. Between 1970 and 1991, prevalence of angina nearly tripled for men over 75 and has continued to rise.

Conclusion Mortality from CVD has declined over the past 50 years, but striking geographic inequalities have remained. Incidence of two major CVD conditions have declined, but continuing increases in prevalence and an ageing population mean that the burden of CVD is still a major issue for the UK.

\section{P1-94 THE EPIDEMIOLOGY AND COSTS OF ANKLE INJURIES: A REVIEW OF THE LITERATURE}

doi:10.1136/jech.2011.142976c.87

I A Bielska, * A Johnson. Queen's University, Kingston, Ontario, Canada

Introduction Ankle sprains are one of the most common injuries presenting to emergency departments, representing $3 \%$ to $5 \%$ of all visits in the UK, and $10 \%$ of all injury-related visits in the USA. Ankle injuries have significant physical and economic consequences for the affected individuals.

Objectives To describe the epidemiology of ankle sprains and fractures among the general population; and to determine the direct and indirect costs related to the diagnosis and treatment of ankle injuries.

Methods A comprehensive literature review of Ovid MEDLINE, EMBASE, Cochrane DSR, ACP Journal Club, AMED, Ovid Healthstar, and CINAHL was conducted for English-language studies on ankle sprains and fractures published from 1980 to 2010.

Results The search identified 2394 studies of which 47 were selected for analysis. A majority of the studies were published in the last decade. The incidence of ankle sprains was 2 to 7 per 1000 personyears, while the incidence of ankle fractures was 1 per 1000 personyears. The costs of emergency ankle sprain management ranged from 126.13 to 2356.21 per patient (2009 CAD), depending on sprain severity. The management costs were higher for ankle fractures: 1692.82 to 15802.26 (2009 CAD) per patient. The economic evaluations were conducted from the societal or healthcare system perspective.

Conclusions Information on the epidemiology of ankle sprains and fractures may help plan for health policy and the provision of health services. Moreover, the cost data may inform future studies undertaking economic evaluations of the diagnosis and treatment of ankle injuries.

\section{P1-95 IS CURRENT POLICY FOR ANAEMIA PREVENTION IN BEDOUIN TODDLERS IN THE NEGEV APPROPRIATE?}

doi:10.1136/jech.2011.142976c.88

\footnotetext{
${ }^{1,2} \mathrm{~N}$ Bilenko, ${ }^{* 1,3} \mathrm{D}$ Fraser, ${ }^{4} \mathrm{M}$ Tatikashvili, ${ }^{2,3}$ I Belmaker. ${ }^{1}$ Department of Epidemiology and Health Services Evaluation, Ben-Gurion University of the Negev, Beer-Sheva, Israel; ${ }^{2}$ Regional Office of Ministry of Health, Southern Region, Beer-Sheva, Israel; ${ }^{3}$ S. Daniel Abraham International Center for Health and Nutrition, Ben-Gurion University of
}

the Negev, Beer-Sheva, Israel; ${ }^{4}$ Soroka University Medical Center, Beer-Sheva, Israel; ${ }^{5}$ Community Health Division, Ben-Gurion University of the Negev, Beer-Sheva, Israel

Introduction Iron deficiency anaemia still affects a quarter of the world's population increasing risk of infectious disease morbidity, impaired growth and mental development. According to current policy children undergo screening for iron deficiency anaemia at age 9-12M. Methods Prospective Study The study population included Moslem Bedouin 2.5-3-Y-old children that followed from 6M in Well Baby Clinic. All parents of participants were interviewed during enrolment and monthly meetings. The blood samples were taken from children at enrolment and during last follow-up visit. Anaemia $(\mathrm{Hb}<11 \mathrm{~g} / \mathrm{dl})$ and Iron Deficiency Index (at least 2 of 6 abnormal indicators, including Haemoglobin, Haematocrit, Mean Corpuscular Volume, Red blood cell distribution Width, serum ferritin, and transferin saturation) were defined.

Results The study population included 180 infants. The mother's young age was found as a risk factor for mild anaemia. Male sex was associated with a higher rate of moderate anaemia compared with female sex $(76.2 \%$ and $52.8 \%$, respectively, $p=0.043)$. Children with anaemia had lower average of dietary iron consumption than children with normal levels of $\mathrm{Hb}(\mathrm{p}=0.009)$. Iron deficiency anaemia at age $6 \mathrm{M}$ was a significant and independent risk factor for toddlers' anaemia $(\mathrm{OR}=3.47, \mathrm{p}<0.001)$ controlling for the mother's age, child gender and consumption of dietary iron

Conclusion The most significant factor for anaemia among this population is iron deficiency anaemia at the age of $6 \mathrm{M}$. Prevention, early detection (at age 6M) and appropriate treatment of anaemia in the first year of life are critical to prevent anaemia and its consequences later life.

\section{P1-96 PRIMARY BONE CANCER IN 0-49 YEAR OLDS IN GREAT BRITAIN, 1980-2005 AND FLUORIDE IN DRINKING WATER: A CASE OF INEQUALITIES?}

doi:10.1136/Jech.2011.142976c.89

${ }^{1} \mathrm{~K}$ Blakey, ${ }^{*} \mathrm{R}$ Feltbower, ${ }^{2} \mathrm{R}$ Parslow, ${ }^{1} \mathrm{P}$ James, ${ }^{1} \mathrm{~B}$ G Pozo, ${ }^{3} \mathrm{C}$ Stiller, ${ }^{3} \mathrm{~T}$ Vincent, ${ }^{3} \mathrm{P}$ Norman, ${ }^{2} \mathrm{P}$ McKinney, ${ }^{3} \mathrm{M}$ Murphy, ${ }^{5} \mathrm{~A}$ Craft, ${ }^{1} \mathrm{R}$ McNally. ${ }^{1}$ Institute of Health 8 Society, Newcastle University, Newcastle-upon-Tyne, UK; ${ }^{2}$ Paediatric Epidemiology Group, University of Leeds, Leeds, UK; ${ }^{3}$ Childhood Cancer Research Group, Department of Paediatrics, University of Oxford, Oxford, UK; ${ }^{4}$ School of Geography, University of Leeds, Leeds, UK; ${ }^{5}$ Northern Institute of Cancer Research, Newcastle University, Newcastle-upon-Tyne, UK

Introduction Primary bone cancers (PBC) occur most often in young people. Osteosarcoma and Ewing sarcoma family of bone tumours (ESFT) are most commonly diagnosed in children but aetiology remains unclear. Fluoride has been proposed as a potential causal agent for $\mathrm{PBC}$. The study investigated whether incidence of PBC was linked with fluoride in drinking water.

Method Incidence data on cases aged $<50$ years diagnosed during 1980-2005 were obtained from all ten regional cancer registries in Great Britain (GB). These data were combined with small-area population census, digital boundary and fluoride monitoring data. Negative binomial regression was used to examine the relationship between incidence rates and census small-area fluoride levels. These models were fitted to small-area census data aggregated into three age bands and by gender with the logarithm of the 'at risk' population as an offset.

Results There were 2566 osteosarcoma cases aged 0-49 years: 817 aged $0-14 ; 1315$ aged $15-29$ and 434 aged $30-49$ years. For ESFT there were 1650 cases aged $0-49$ years: 659 aged $0-14 ; 800$ aged 15-29 and 191 aged 30-49 years. After adjustment for age and gender, no statistically significant association was found between osteosarcoma or ESFT and fluoride: RR for one part per million increase in fluoride level $=0.993$; $95 \%$ CI 0.843 to 1.171 and 0.860 ; 95\% CI 0.696 to 1.064 respectively. 